\title{
Sumoylation of Rapl mediates the recruitment of TFIID to promote transcription of ribosomal protein genes
}

\author{
Pierre Chymkowitch, ${ }^{1,2}$ Aurélie Nguéa P, ${ }^{1,2,5}$ Håvard Aanes, ${ }^{1,2,5}$ Christian J. Koehler, ${ }^{3}$ \\ Bernd Thiede, ${ }^{3}$ Susanne Lorenz, ${ }^{4}$ Leonardo A. Meza-Zepeda, ${ }^{4}$ Arne Klungland, ${ }^{1,2}$ \\ and Jorrit M. Enserink ${ }^{1,2}$ \\ ${ }^{7}$ Institute of Microbiology, Clinic for Diagnostics and Intervention, Oslo University Hospital, N-0027 Oslo, Norway; ${ }^{2}$ University \\ of Oslo, 0316 Oslo, Norway; ${ }^{3}$ The Biotechnology Centre of Oslo, University of Oslo, 0349 Oslo, Norway; ${ }^{4}$ Department of Tumor \\ Biology, The Norwegian Radium Hospital, and Genomics Core Facility, Oslo University Hospital, NO-0310 Oslo, Norway
}

\begin{abstract}
Transcription factors are abundant Sumo targets, yet the global distribution of Sumo along the chromatin and its physiological relevance in transcription are poorly understood. Using Saccharomyces cerevisiae, we determined the genome-wide localization of Sumo along the chromatin. We discovered that Sumo-enriched genes are almost exclusively involved in translation, such as tRNA genes and ribosomal protein genes (RPGs). Genome-wide expression analysis showed that Sumo positively regulates their transcription. We also discovered that the Sumo consensus motif at RPG promoters is identical to the DNA binding motif of the transcription factor Rapl. We demonstrate that Rapl is a molecular target of Sumo and that sumoylation of Rapl is important for cell viability. Furthermore, Rapl sumoylation promotes recruitment of the basal transcription machinery, and sumoylation of Rapl cooperates with the target of rapamycin kinase complex 1 (TORCl) pathway to promote RPG transcription. Strikingly, our data reveal that sumoylation of Rapl functions in a homeostatic feedback loop that sustains RPG transcription during translational stress. Taken together, Sumo regulates the cellular translational capacity by promoting transcription of tRNA genes and RPGs.
\end{abstract}

[Supplemental material is available for this article.]

Nutrient availability is a major challenge to cellular homeostasis. Changes in nutrient availability activate signal transduction pathways that rewire cell metabolism and mobilize new energy sources (De Virgilio and Loewith 2006). The rapamycin-sensitive target of rapamycin complex 1 (TORC1) is a kinase complex that coordinates the cellular nutrient response (Loewith et al. 2002). Under nutrient-rich conditions, TORC1 inhibits catabolism while promoting anabolic processes like protein synthesis (De Virgilio and Loewith 2006).

The rate of protein synthesis is dependent on the rate of ribosome synthesis, which requires transcription of ribosomal protein genes (RPGs) (De Virgilio and Loewith 2006). RPGs are among the most intensively transcribed genes; $50 \%$ of all RNA polymerase II (RNAPII) initiation events occur at the 138 RPG promoters in the yeast genome (Warner 1999). The promoter-bound transcription factor Rap1 plays a key role in transcription of nearly all RPGs. Rap1 is required for recruitment of Ifh1, Fhl1, Hmo1, Sfp1, and TFID, which promote recruitment of RNAPII (Schawalder et al. 2004; Wade et al. 2004; Garbett et al. 2007; Knight et al. 2014). Additionally, Rap1 mediates transcription of about 180 nonRPGs, including glycolytic genes (Lieb et al. 2001), and it binds telomeric regions to regulate telomere length and to maintain a repressed chromatin state (Shore 1997).

\footnotetext{
${ }^{5}$ These authors contributed equally to this work.
} Corresponding author: jorrit.enserink@rr-research.no Article published online before print. Article, supplemental material, and publication date are at http://www.genome.org/cgi/doi/10.1101/gr.185793.114. Freely available online through the Genome Research Open Access option
RPG transcription is strongly dependent on nutrient availability, and TORC1 activates RPG transcription by promoting phosphorylation of Sfp1 and Ifh1, leading to their recruitment to RPG promoters (Schawalder et al. 2004; Wade et al. 2004; Cai et al. 2013). Inhibiting TORC1 with rapamycin results in rapid dephosphorylation and release from the promoter of Sfp1 and Ifh1, thereby inhibiting transcription of RPGs. In contrast, localization of Rap1 to RPG promoters is not affected by TORC1 (Wade et al. 2004). Despite the fact that localization of Rap1 to chromatin is dynamic (Lickwar et al. 2012), it is currently not known whether the cell regulates the transcriptional activity of Rap1 (Schawalder et al. 2004).

Nutrient starvation elicits a stress response in yeast (Simpson and Ashe 2012), and stress induces sumoylation of various proteins (Zhou et al. 2004; Tempe et al. 2008; Jentsch and Psakhye 2013). Proteomic studies have found that transcription factors (TFs) are major Sumo substrates, although the functional consequences of most sumoylation events remain unknown (Wohlschlegel et al. 2004; Hannich et al. 2005). Current consensus is that sumoylation of TFs generally represses transcription (Gill 2005). For instance, sumoylation of the repressor complex Tup1Ssn6 inhibits transcription (Texari et al. 2013). Furthermore, sumoylation of histones inhibits transcription, although the physiological relevance remains unknown (Nathan et al. 2006). Recent studies indicate that Sumo can also have a positive role (Rosonina et al. 2010). Surprisingly, a comprehensive analysis of the genome-

(C) 2015 Chymkowitch et al. This article, published in Genome Research, is available under a Creative Commons License (Attribution-NonCommercial 4.0 International), as described at http://creativecommons.org/licenses/by-nc/4.0/. 
wide association of Sumo with chromatin has not yet been performed in S. cerevisiae, and the physiological relevance of sumoylation of the vast majority of TFs remains unclear.

In this study, we analyze the genome-wide localization of Sumo on chromatin and describe a molecular mechanism by which Sumo promotes transcription of RPGs.

\section{Results}

\section{Genome-wide analysis of Sumo reveals that Sumo localizes} to genes involved in translation

To better understand the function of Sumo in transcription, we performed Sumo ChIP-seq experiments with a strain expressing FLAG-tagged SMT3 (the yeast gene encoding Sumo), using untagged cells as a negative control (Supplemental Fig. S1A). We identified 670 significant Sumo peaks corresponding to 631 unique ORFs (Fig. 1A; Supplemental Table S1, tab1), including several genes previously found to be enriched for Sumo, such as CDC19, $A D H 1, P M A 1$, and the GAL1/10 promoter, but not ACT1 (Fig. 1A; Supplemental Fig. S1B; Rosonina et al. 2010; Texari et al. 2013). $\mathrm{GO}$ analysis showed strong overrepresentation of genes involved in mRNA translation (Fig. 1B; Supplemental Table S2). However, visual inspection of this data set revealed that the peak calling software had inaccurately assigned several Sumo peaks to dubious ORFs that were in close proximity to genuine ORFs (Supplemental Fig. S1C). Using more stringent filters with a higher peak-calling cutoff score, combined with visual inspection of all the Sumo peaks (see Methods), we exchanged these dubious ORFs with the genuine Sumo-containing ORFs. Although this resulted in removal of several genes with low levels of Sumo, including CDC19, PMA1, and
ADH1 (Supplemental Table S1, tab2), we decided to focus on this stringent data set for the rest of our study, accepting that higher confidence comes at the cost of decreased sensitivity. This highconfidence Sumo data set contained 423 Sumo peaks at 395 unique genes, and consisted of 246 RNAPIII-transcribed tRNA genes, 110 RPGs, 12 protein-coding genes, and 27 noncoding RNAs, like snoRNAs (Fig. 1C; Supplemental Table S1). Together, these data show that in log-phase cells, RNAPIII-transcribed genes and RPGs are major targets of Sumo.

Metagene analysis of Sumo localization patterns revealed that RPGs had a strong Sumo peak several hundred base pairs upstream of the transcription start site (TSS), followed by a significant amount of Sumo toward the $5^{\prime}$ end of the ORF, whereas the RNAPIII-transcribed tRNA genes had a single, sharp Sumo peak right at the TSS (Fig. 1D).

The genome-wide results were confirmed by targeted ChIPqPCR on selected genes (Fig. 1E). We also performed these experiments in the temperature-sensitive $u b c 9-1$ mutant, which expresses a destabilized form of the E2 conjugase Ubc9, resulting in a strong reduction in cellular protein sumoylation even at a permissive temperature of $30^{\circ} \mathrm{C}$ (Supplemental Fig. S1D; Betting and Seufert 1996; Rosonina et al. 2010). We carried out these experiments at $30^{\circ} \mathrm{C}$, because the restrictive temperature of $37^{\circ} \mathrm{C}$ causes a stress response accompanied with increased global sumoylation (Tempe et al. 2008), which could confound our results. Importantly, Sumo levels at the promoter of RPL35A and at $t D N A^{W}$ (which encodes tRNA-tryptophan, $t R N A^{W}$ ) were dramatically reduced in this mutant (Supplemental Fig. S1E), and this reduction was fully rescued by a plasmid containing WT UBC9 (Supplemental Fig. S1F), demonstrating that our data are specific and not artifacts caused by "hyper-ChIPable" chromatin (Teytelman et al. 2013).
A
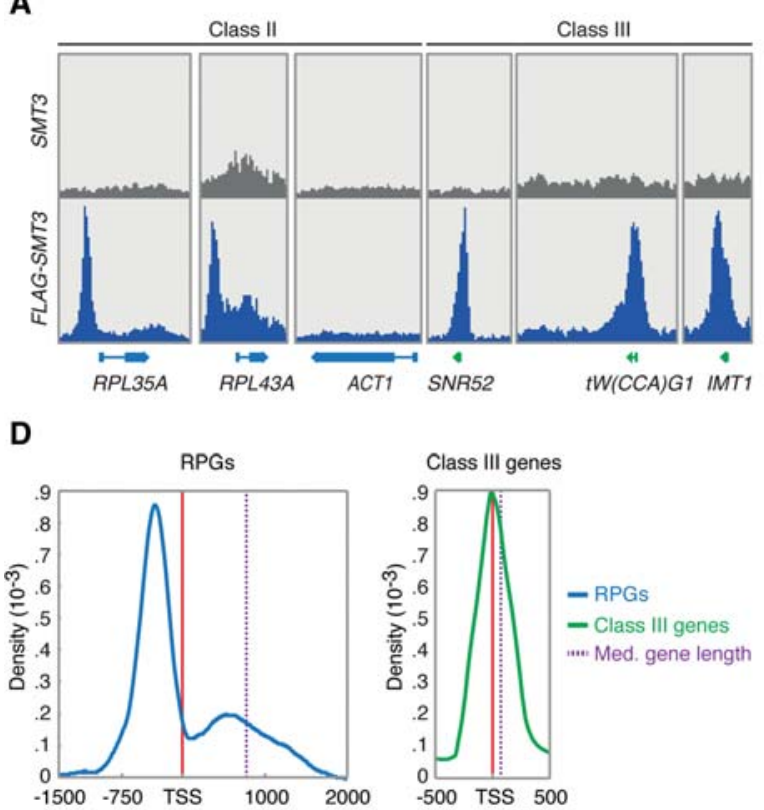

B

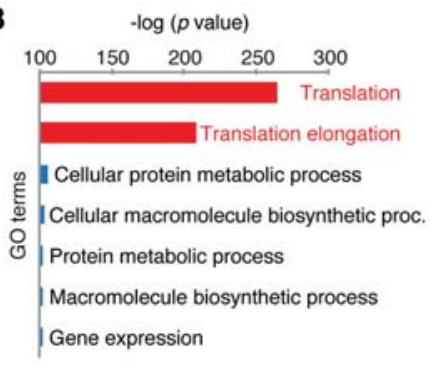

E

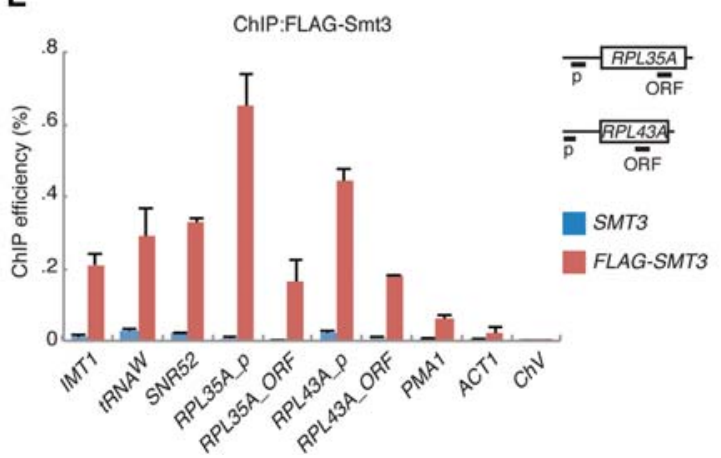

Figure 1. Sumo is enriched at class III genes and RPGs. (A) Snapshots of the Sumo ChIP-seq experiment with FLAG-tagged Sumo (FLAG-SMT3) and untagged control cells (SMT3). $(B, C)$ GO analysis of the ChIP-seq data set reveals highly significant overrepresentation of genes involved in translation, such as RPGs and class III genes. $(D)$ Metagene analysis of Sumo distribution patterns at RPGs and class III genes. $(E)$ Validation of ChIP-seq data by conventional ChIP-qPCR. Error bars indicate SEM of three independent experiments.

\section{Genome Research}

www.genome.org 
In conclusion, these results show that Sumo primarily localizes to class III genes and RPGs.

\section{TORCl and Sumo pathways cooperate to promote transcription of RPG and tRNA genes}

To test whether Sumo is important for transcription, we performed RNA-seq experiments using wild-type (WT) and ubc9-1 cells (Supplemental Table S3). Globally, the relative expression levels of the transcriptome were unaffected in the $u b c 9-1$ mutant (Fig. 2A). In sharp contrast, expression of almost all RPGs was reduced in ubc9-1 mutants (Fig. 2B; Supplemental Fig. S2A). These data show that Ubc9 positively regulates the expression of RPGs.

tRNA genes and RPGs are regulated by TORC1 (Lempiainen and Shore 2009; Wei and Zheng 2010); therefore, we hypothesized that Ubc9 cooperates with TORC1 to regulate their transcription. We performed whole-transcriptome analysis of WT and $u b c 9-1$ cells after treatment with the TORC1-specific inhibitor rapamycin. No effect was observed on global transcription (Fig. 2A; Supplemental Fig. S2B). As expected, transcription of RPGs was specifically inhibited by rapamycin; however, RPG transcription was significantly further reduced in rapamycin-treated $u b c 9-1$ mutants (Fig. 2B). The RNA-seq data were validated by targeted qPCR exper- iments, which also included the tRNAs IMT1, and $t R N A^{W}$ (Fig. 2C). These experiments confirm our ChIP-seq data and show that TORC1 and Ubc9 have additive effects on transcription of tDNA and RPGs. In contrast, rapamycin did not affect PMA1 expression, and the $u b c 9-1$ mutation had only a relatively minor effect on expression of PMA1, which is consistent with our finding that Sumo levels are relatively low at this gene (Supplemental Fig. S1B). Furthermore, expression of $A C T 1$, which is neither controlled by TORC1 nor enriched for Sumo (Fig. 1A), was completely unaffected by rapamycin and the $u b c 9-1$ mutation (Fig. 2C). These data demonstrate that TORC1 and Ubc9 cooperate specifically in transcription of tRNA genes and RPGs.

To determine whether TORC1 affects sumoylation at chromatin, we performed a Sumo ChIP-seq experiment in WT and $u b c 9-1$ cells treated with rapamycin. Because TORC1 is known to promote transcription of RPGs and tRNA genes, and because we found that Sumo also positively affects transcription of these genes, we expected that rapamycin treatment would either have no effect or that it would lead to decreased Sumo levels. Indeed, rapamycin caused decreased sumoylation at tRNA genes in WT cells and a further decrease in $u b c 9-1$ mutants (Fig. 2D,E). However, contrary to our expectations, rapamycin treatment resulted in increased Sumo levels at the promoter of RPGs but decreased sumoylation
A

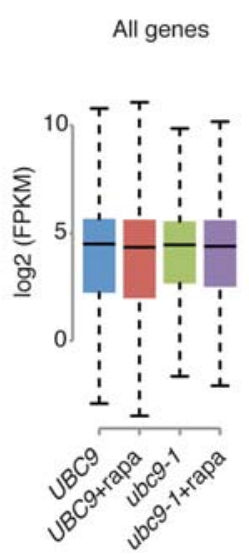

D
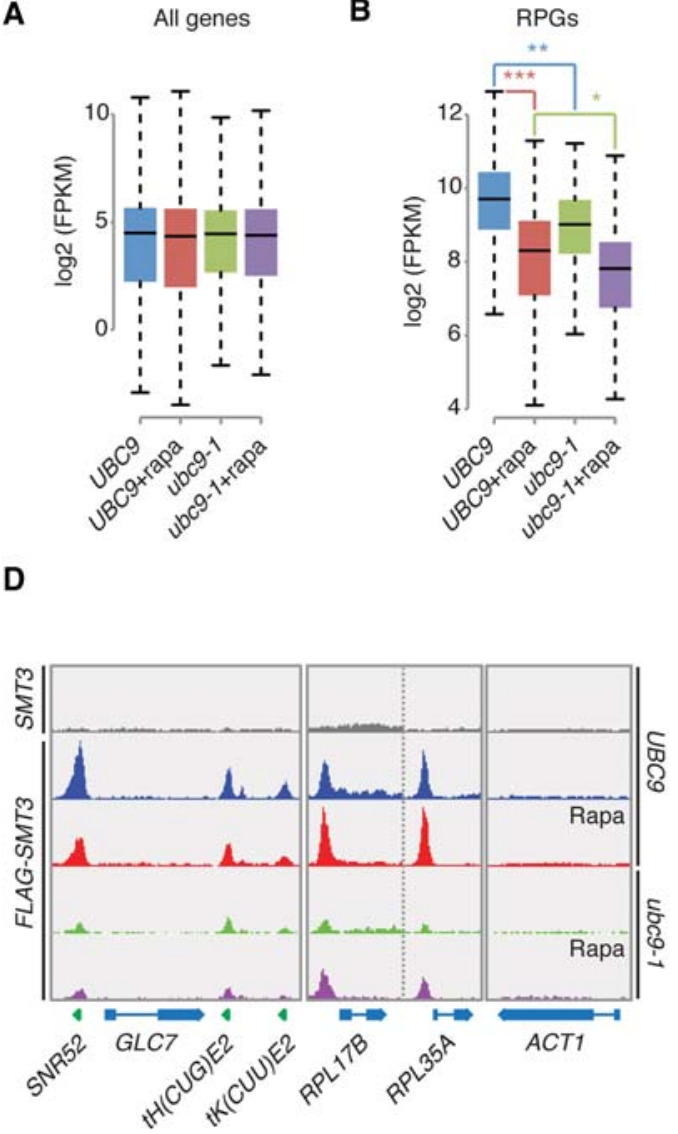

C

E
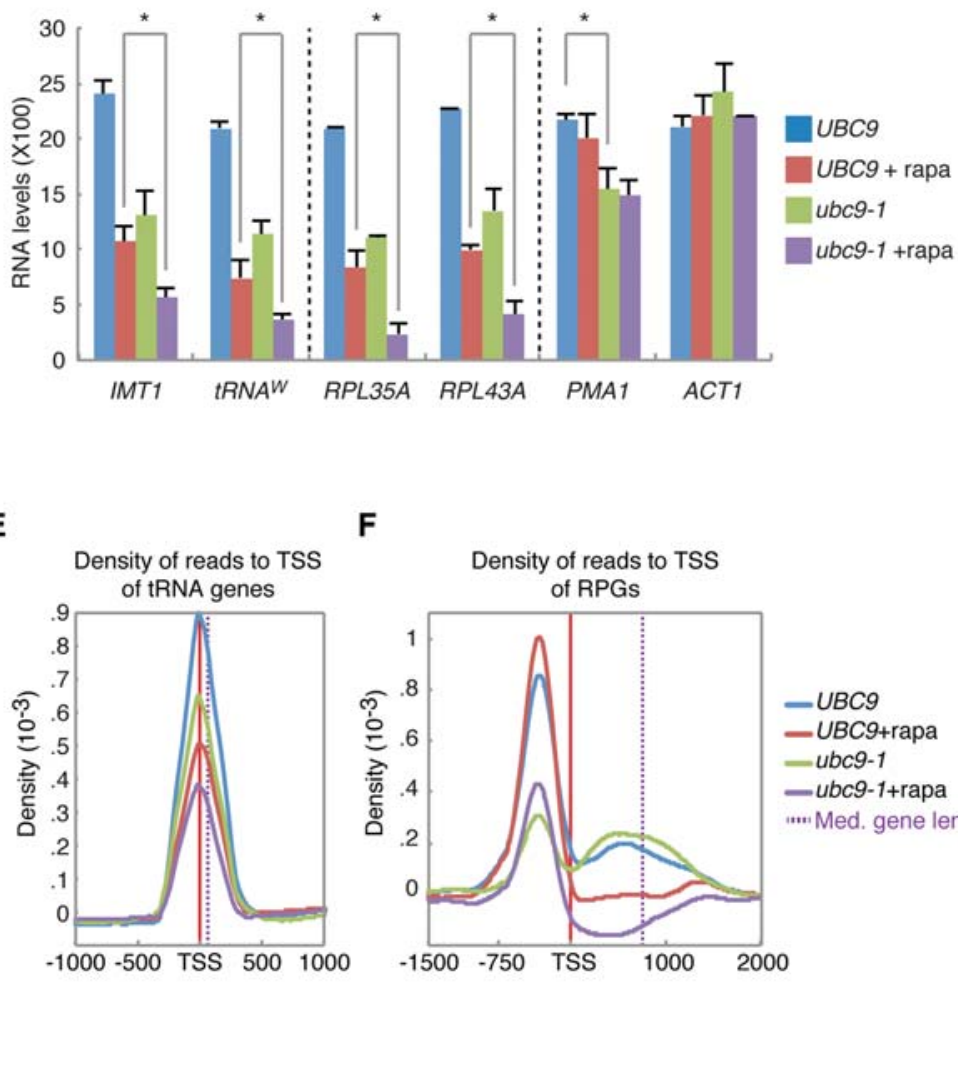

$\mathbf{F}$

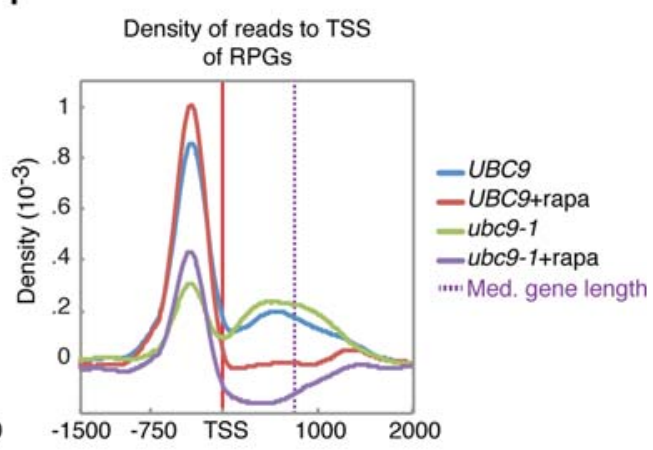

Figure 2. Ubc9 and TORC1 cooperate in regulation of transcription. (A) The global transcriptome is not affected by either Ubc9 or TORC1. WT cells and ubc9-1 mutants were treated for $30 \mathrm{~min}$ with $100 \mathrm{nM}$ rapamycin (rapa) or with DMSO before RNA-seq. Potential differences between groups were tested with a two-sided Mann-Whitney $U$ test. (B) Transcription of RPGs is regulated by both TORC1 and Ubc9. Cells were treated and RNA analyzed as in $A$. Differences between groups were tested as in $A .\left(^{*}\right) P=0.008192 ;\left(^{* *}\right) P=7.182 \times 10^{-6} ;\left(^{* * *}\right) P=1.691 \times 10^{-15}$. (C) Targeted qPCR experiment. Error bars, SEM of three independent experiments. $\left(^{*}\right) P<0.01$. (D) Snapshots of Sumo ChIP-seq in WT cells and ubc9-1 mutants treated with DMSO or rapamycin. $(E, F)$ Metagene analysis of Sumo patterns at tDNA $(E)$ and RPGs $(F)$. 
in the body of the genes (Fig. 2D,F). This rapamycin-dependent pattern was similar in $u b c 9-1$ mutants, although the overall level of Sumo was reduced. These ChIP-seq data were validated by targeted ChIP-qPCR, confirming that TORC1 differentially affects the sumoylation patterns of RPGs and tRNA genes (Supplemental Fig. S2C).

Finally, we studied the dynamics of Sumo levels at tRNA genes and RPGs during rapamycin treatment and found that Sumo levels at $t D N A^{W}$ rapidly decreased upon rapamycin treatment, which resulted in a reduction of $t R N A^{W}$ levels (Supplemental Fig. S2D). In contrast, Sumo levels at the promoter region of RPL35A progressively increased, whereas RPL35A expression levels were negatively affected by rapamycin (Supplemental Fig. S2E).

Taken together, these data reveal a dynamic function for TORC1 and Ubc9-Sumo in transcription of RPGs and tRNA genes. The data also reveal that although both TORC1 and Sumo are important for expression of RPGs (Fig. 2B), inhibition of TORC1 results in a paradoxical increase in Sumo levels at RPG promoters (Fig. 2F; Discussion).

\section{Sumoylation at RPGs occurs at Rapl binding sites}

To gain insight into the mechanism by which Sumo regulates transcription, we determined whether any particular DNA sequence motif occurred within peaks of Sumo enrichment using our Sumo-ChIP-seq data set (Fig. 3A, top). When we compared this sequence to known TF consensus motifs, we found that it corresponded to the consensus sequence of the transcription factor Rap1 (Fig. 3A, bottom). Interestingly, several reports have indicated that Rap1 may be a Sumo target, although its relevance for transcription was never explored (Wohlschlegel et al. 2004; Denison et al. 2005; Hang et al. 2011; Lickwar et al. 2012; Albuquerque et al. 2013; Lescasse et al. 2013). Encouraged by these findings, we decided to focus on sumoylation of Rap1 at RPG promoters.

First, we performed a Rap1 ChIP-seq experiment (Supplemental Table S4) and found excellent correlation with a previous study that mapped Rap1 (overlap of 424 out of 429 genes) (Lickwar et al. 2012). The Rap1 binding sites were then aligned with the genomic sites enriched for Sumo. Strikingly, 95\% of RPGs showed perfect colocalization of promoter-bound Rap1 and Sumo (Fig. 3B,C). A small number of RPGs does not contain a Rap1 consensus motif (Lieb et al. 2001). Our ChIP-seq data showed that Sumo was undetectable at RPGs that did not recruit Rap1, including RPL18B (Fig. 3B; Supplemental Fig. S3A). The presence of Sumo at the Rap1 binding site was specific for RPGs, because non-RPGs that also recruit Rap1, like ATG19 (Lieb et al. 2001), either did not contain any Sumo (Fig. 3B) or they had a Sumo peak that did not align with the Rap1 peak (Fig. 3D; Supplemental Fig. S3B), such as SNR47 (Supplemental Fig. S3C). This indicates that other TFs must be sumoylated at these genes, which is the scope of future studies. Taken together, these data strongly suggest that Rap1 is a Sumo target at RPG promoters.

To demonstrate that Rap1 is essential for sumoylation at RPG promoters, we performed Rap1 depletion experiments. The endogenous RAP1 promoter was replaced with the GAL1 promoter, which is active in the presence of galactose but rapidly repressed when cells are transferred to glucose. Indeed, incubation in glucose resulted in depletion of Rap1 and its disappearance from the promoter of $R P L 35 A$ (Fig. 3E), strongly decreasing RPL35A mRNA levels (Supplemental Fig. S3D). Strikingly, Sumo also disappeared from this region with kinetics indistinguishable from those of Rap1 (Fig. 3E), showing that Rap1 is required for sumoylation at RPG promoters. Indeed, mutating the Rap1 binding site at the promoter of RPS19B prevented the recruitment of Sumo (Fig. 3F), whereas recruitment of Sumo at RPL35A (with intact Rap1 binding sites) as well as SCR1 (an RNAPIII-transcribed gene which recruits Sumo independently of Rap1) was unaffected. Together, these data demonstrate that the presence of Sumo at RPG promoters specifically depends on the presence of Rap1.

\section{Sumoylation of Rapl is required for transcription of RPGs}

Next, we wanted to identify targets of Sumo with an unbiased experimental approach. Using HIS $_{6}$-FLAG-tagged Sumo, we first purified Sumo from formaldehyde-fixed cells with FLAG antibodies (semi-native conditions) (Supplemental Fig. S3E) or from cells lysed with TCA using the $\mathrm{HIS}_{6}$ tag (denaturing conditions; see Methods). Analysis of the eluates by mass spectrometry (MS) showed that Rap1 was present in the Sumo-containing protein fractions (Supplemental Table S5). We also performed this analysis with cells treated with DMSO or rapamycin and found that the presence of Rap1 in the Sumo-containing protein fractions was more pronounced upon treatment with rapamycin (Supplemental Fig. S3F). To directly show that Rap1 is sumoylated, $\mathrm{HIS}_{6}$-tagged Sumo was purified under denaturing conditions followed by Western blotting with Rap1-specific antibodies. This confirmed that Rap1 is a Sumo target and that Rap1 sumoylation increases after rapamycin treatment (Fig. 4A), whereas total Rap1 levels remained unchanged (Supplemental Fig. S3G). As a control, rapamycin did not affect sumoylation of the septin Cdc11, a well-known Sumo target (Johnson and Blobel 1999). Similar data were obtained in a reciprocal experiment in which purified $\mathrm{HIS}_{11^{-}} \mathrm{Myc}_{2^{-}}$ tagged Rap1 was analyzed by Western blotting with Sumo antibodies (Fig. 4B). These data demonstrate that Rap1 is a direct target of Sumo and that its sumoylation status increases upon inhibition of TORC1.

Recently, sumoylation of lysines K240 and K246 in Rap1 ("Rap1-K2R") (see Fig. 4C for an overview of rap1 mutants used in this study) was shown to be important for telomere homeostasis (Lescasse et al. 2013). However, we found that mutation of these sites did not affect Rap1 sumoylation (Fig. 4B), indicating that other lysines must be sumoylated. Sumoylation-site prediction software (Zhao et al. 2014) predicted a total of nine sumoylation sites (K43, K117, K118, K240, K246, K309, K462, K463, and K651). We mutated these nine lysines to arginine ("rap1-K9R") and found that many sumoylation events were almost completely prevented (note that this mutant migrates slightly faster than WT Rap1) (Fig. 4B). We also studied the growth rate of this mutant using the aforementioned Rap1 depletion strain, in which HA-tagged $R A P 1$ is expressed from the GAL1 promoter. This depletion strain was transformed with a construct harboring various GFP-tagged rap1 mutants under control of the glucose-inducible GPD promoter. As shown in Figure 4D, only expression of the rap1-K9R mutant caused a growth defect at $30^{\circ} \mathrm{C}$ and inviability at $37^{\circ} \mathrm{C}$. In an attempt to narrow down the sumoylation sites, we made three additional rap1 mutants (rap1-K651R, rap1-K(3N)R, and rap1-K(3C)R), but none of these mutants displayed a detectable growth defect (Fig. 4D). These data indicate that Rap1 is sumoylated on multiple lysines with partially redundant functions.

We studied the effect of these rap1 mutants on RPG expression using the Rap1 depletion system. Cells were grown to log phase in galactose and transferred to glucose-containing medium

\section{Genome Research}

www.genome.org 
A

\section{"Sumo motif" (ChIP-seq, $p=\mathrm{e}^{-56}$ ) ACACCCATACAT CACCCATACA}

Rap1 motif (Database)

\section{C}

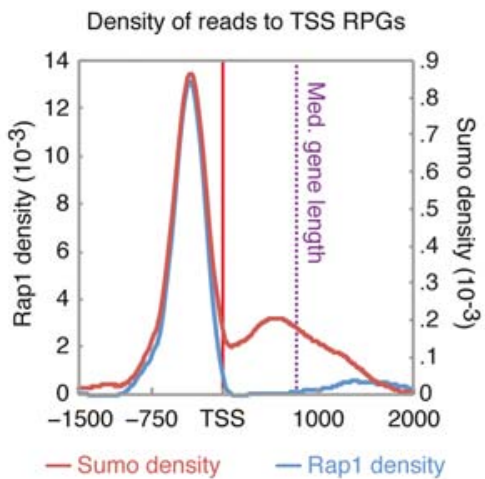

B

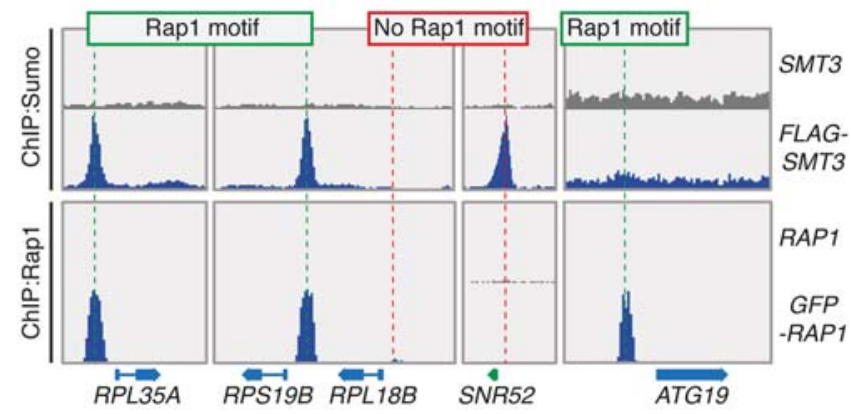

D

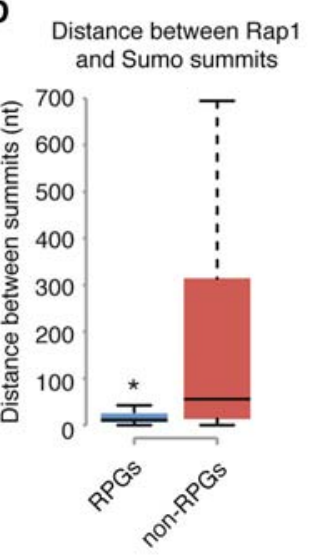

E
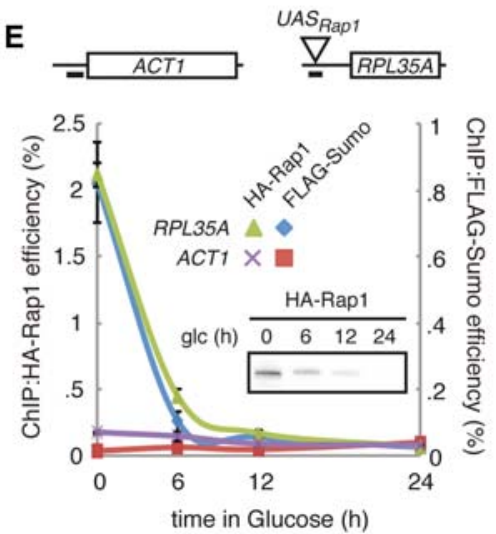

UAS Rap1

UAS $_{\text {Rap1 }}$

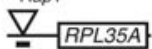

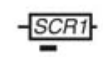

RPS19B UAS Rap1

RPS19B UAS Rap $1:$ SS

Figure 3. Sumoylation at RPG promoters requires the presence of Rap1. (A) The Sumo consensus motif is highly similar to the Rap1 DNA binding motif. (B) Snapshots of the Rap1 and Sumo ChIP-seq experiments. (C) Perfect overlap between Sumo peaks and Rap1 peaks at RPG promoters. (D) Box plot showing the nucleotide (nt) distance between Sumo and Rap1 peaks at RPGs and non-RPGs. Median values are 11 and 55.5 nt for RPGs and non-RPGs, respectively (Mann-Whitney $U$ test; $W=3823, P=3.837 \times 10^{-5}$ ). (E) Recruitment of Sumo depends on Rap1. Cells expressing Rap1 from the GAL1 promoter were grown to log phase in the presence of galactose, washed, and incubated in glucose. Levels of endogenous HA-Rap 1 and FLAG-Sumo at the RPL35A promoter were analyzed by ChIP. Primer pair locations are indicated above the graph. (Inset) Level of Rap1 by Western blotting. Error bars, SEM. ( $F$ ) The Rap1 binding site at the RPS19B promoter was either left intact (RPS19B_UAS $\left.{ }_{R A P 1}\right)$ or replaced with scrambled sequence (RPS19B_UAS ${ }_{R A P 1} S S$; see Supplemental Methods for construction details). Insertion of scrambled sequence resulted in loss of Rap1 at RPS19B (left panel) and prevented recruitment of Sumo (right panel). Error bars, SEM.

(Supplemental Fig. S3H), after which the levels of Rap1 and Sumo at the RPL35A and RPL43A promoters were analyzed (Fig. 4E; Supplemental Fig. S3I,J). Interestingly, although Rap1-K9R efficiently localized to RPG promoters (Supplemental Fig. S3J), Sumo levels were diminished and RPG expression was strongly reduced (Fig. 4E,F), whereas little or no effect on RPG expression was observed in rap1-K651R, rap1-K(3N)R, and rap1-K(3C)R mutants (Fig. 4F).

Taken together, these data show that Rap1 sumoylation is important for RPG expression and cell viability.
Sumoylation of Rapl promotes recruitment of the basal transcription machinery

Because recruitment of RNAPII to RPGs depends on the interaction between Rap1 and TFID (Garbett et al. 2007), we hypothesized that sumoylation of Rap1 is important for recruitment of TFIID and RNAPII. We performed an RNAPII ChIP-seq experiment and found that the levels of RNAPII at RPGs were indeed strongly reduced in $u b c 9-1$ mutants and nearly undetectable after rapamycin treatment, whereas RNAPII levels at non-RPGs remained 
A

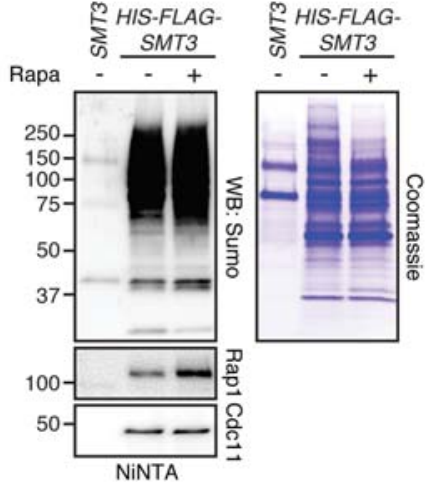

D
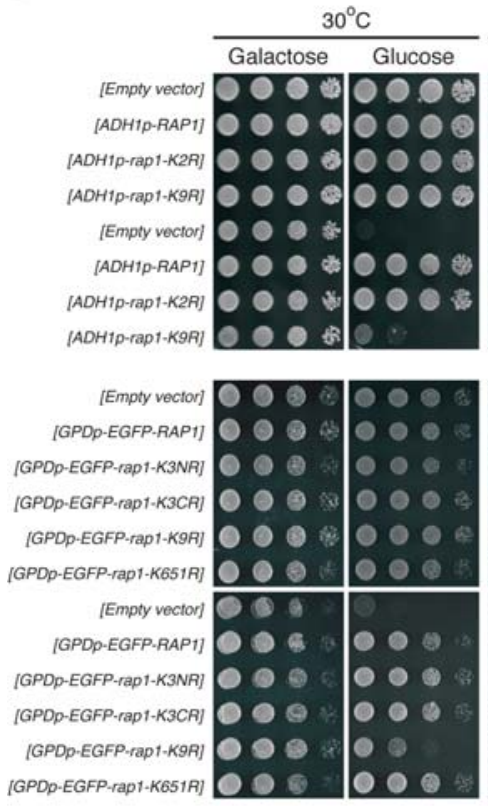

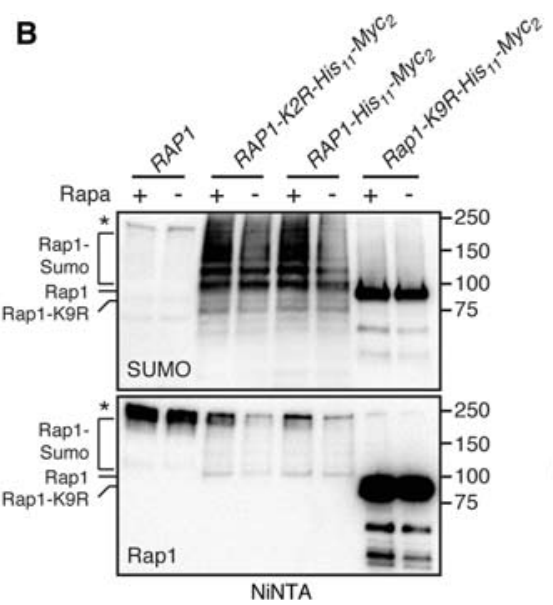

C

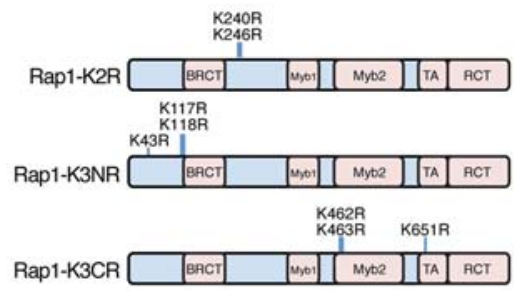

K651R

Rap1-K651R \begin{tabular}{|l|l|l|l|l|l|l|}
\hline & BACT & & yyol & Myb2 & TA & ACT \\
\hline
\end{tabular}

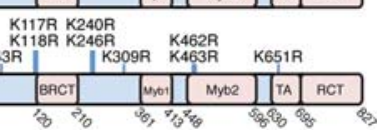

Rap1-K9R

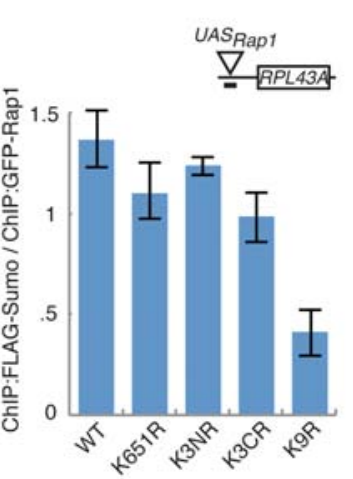

E

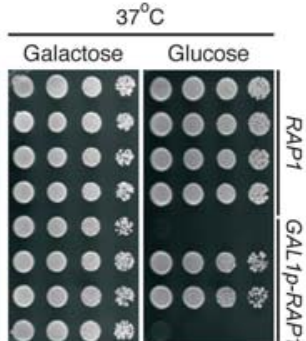

E

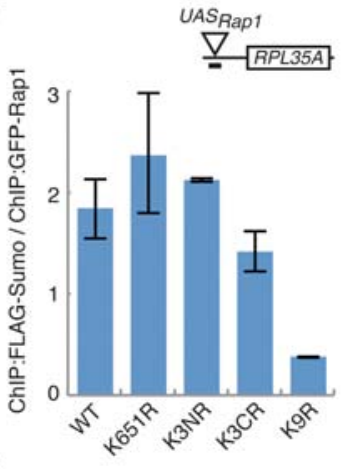

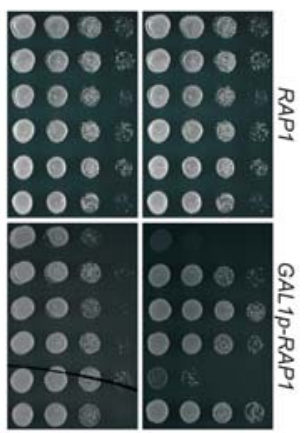

$F$

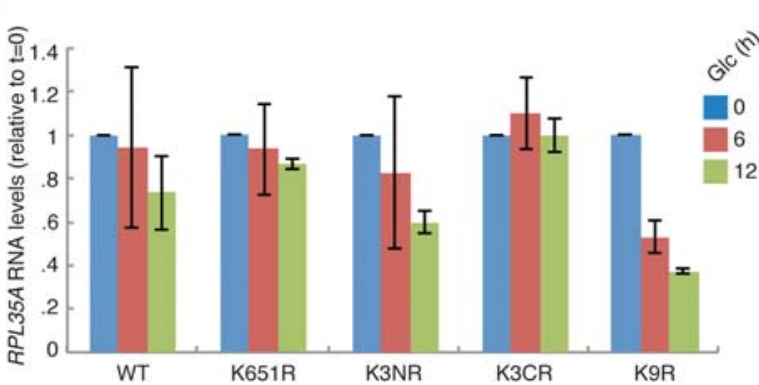

Figure 4. Sumoylation of Rap1 is required for efficient RPG expression. (A) Sumoylation of Rap1 increases after rapamycin treatment. Cells were treated for 30 min with $100 \mathrm{nM}$ rapamycin, and $\mathrm{HIS}_{6}$-FLAG-tagged Sumo was purified under denaturing conditions. Levels of Rap1 and Cdc11 in eluates were analyzed by Western blotting (left). Total protein levels in the eluates were visualized by Coomassie staining (right). (B) WT cells transformed with plasmids harboring $\mathrm{HIS}_{11}-\mathrm{Myc}_{2}$-tagged versions of $R A P 1$, rap1-K2R, or rap1-K9R were treated with $100 \mathrm{nM}$ rapamycin for 30 min. Rap1, Rap1-K2R, and Rap1-K9R were purified under denaturing conditions and visualized by Western blotting with Rap1 antibodies. (C) rap1 mutants used in this study. (D) Expression of rap1-K9R as the sole source of Rap1 results in severe growth defects. Cells expressing RAP1 from its endogenous promoter ("RAP1") or from the GAL1/10 promoter ("GAL1_p-RAP1") were transformed with an empty vector or with plasmids containing either $R A P 1$, rap1-K2R, rap1-K9R, rap1-K3NR, rap1-K3CR, or rap 1-K651R under control of the $A D H 1$ or the GPD promoter, as indicated. Tenfold dilutions were spotted on plates containing either galactose or glucose and incubated for $2 \mathrm{~d}$. $(E, F)$ Rap1 sumoylation is important for transcription. FLAG-Sumo cells expressing endogenous RAP1 from the GAL1/10 promoter were transformed with plasmids harboring wild-type $R A P 1(\mathrm{WT})$ or the rap1-K3NR, K3CR, K651R, or K9R alleles. Cells were grown to log phase in galactose, washed, and incubated in glucose for the indicated times, after which Sumo levels at the RPL35A and RPL43A promoters were analyzed by ChIP $(E)$, and immature (i.e., unspliced) RNA levels of RPL35A were determined by qPCR $(F)$. Error bars, SEM.

unchanged (Supplemental Fig. S4A). These data were confirmed by targeted ChIP-qPCR (Fig. 5A). Interestingly, recruitment of the TFIID component TBP to RPG promoters was also strongly reduced under these conditions, suggesting that Rap1 sumoylation mediates recruitment of TFIID (Fig. 5B). We tested this hypothesis directly by coimmunoprecipitation experiments. Although the TFIID subunit Taf4 efficiently coimmunoprecipitated with HAtagged Rap1 in WT cells, almost no Taf 4 coimmunoprecipitated with HA-Rap1-K9R (Fig. 5C). The interaction between sumoylated Rap1 and TFIID did not depend on TORC1 (Fig. 5D), which is consistent with our previous data indicating that Ubc9 and TORC1 act in parallel pathways. Finally, we analyzed recruitment of TFIID and RNAPII to RPGs in cells expressing nonsumoylatable Rap1$\mathrm{K} 9 \mathrm{R}$ as the sole source of Rap1. In accordance with the data obtained with the $u b c 9-1$ mutant, recruitment of TFID and RNAPII was strongly reduced in these cells (Fig. 5E,F). We conclude that

\section{Genome Research}

www.genome.org 
A
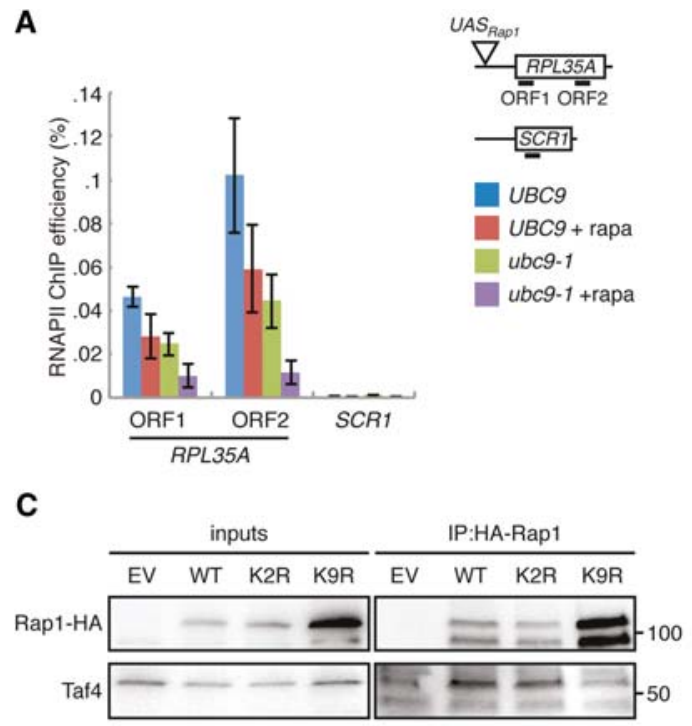

E
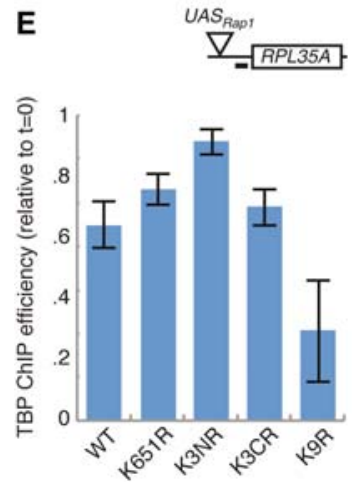
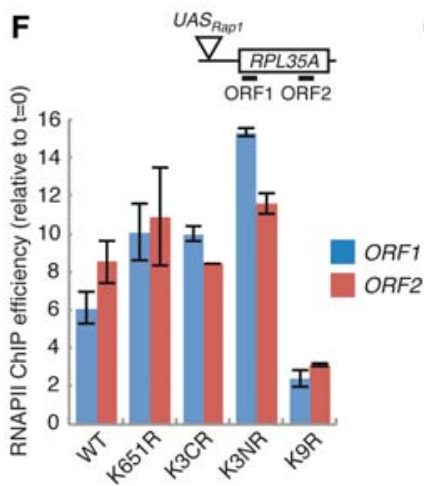

B
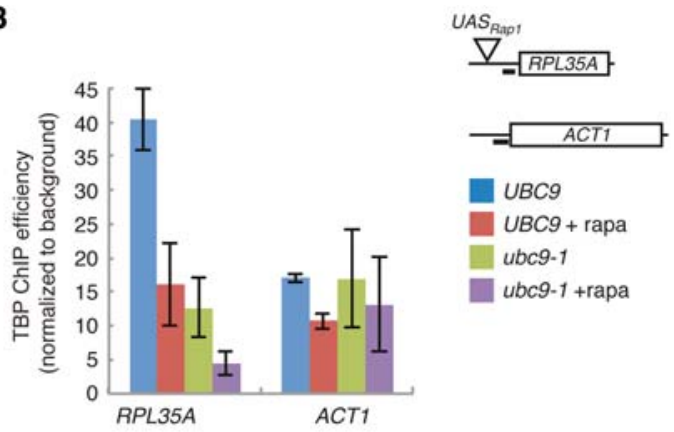

UBC9

UBC9 + rapa

ubc9-1

$u b c 9-1+$ rapa

D

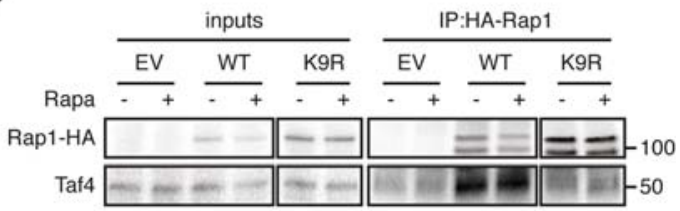

G

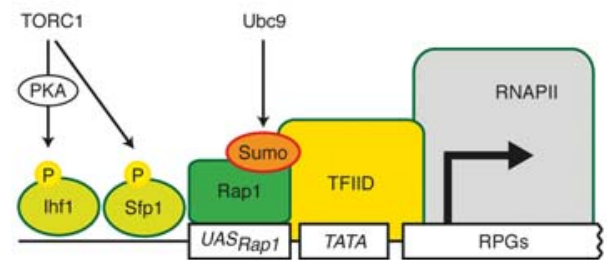

Figure 5. Sumoylation of Rap1 mediates recruitment of the basal transcription machinery. (A) The level of RNAPII at RPL35A depends on the combined activity of TORC1 and Ubc9. Log-phase cells were treated with $100 \mathrm{nM}$ rapamycin for 30 min, after which the level of RNAPII at RPL35A and SCR1 was analyzed by ChIP. The location of primer pairs is indicated. Error bars, SEM. (B) TFIID recruitment depends on TORC1 and Ubc9. Log-phase cells were treated as in A, and the level of TBP at RPL35A and ACT1 was determined by ChIP. Error bars, SEM. (C) Rap1 sumoylation promotes the interaction with TFIID. WT cells transformed with plasmids expressing HA-tagged wild-type RAP1, rap1-K2R, or rap 1-K9R were subjected to immunoprecipitation with anti-HA beads followed by Western blotting with anti-Taf4 antibodies. (D) TORC1 activity does not modulate the interaction between Rap1 and TFIID. Cells treated with $100 \mathrm{nM}$ rapamycin (30 min) were processed as in C. $(E, F)$ Sumoylation of Rap1 promotes recruitment of TFIID and RNAPII to RPL35A. ChIPs were performed as in Figure 4E using antibodies against TBP $(E)$ and RNAPII $(F)$. Error bars, SEM. (G) Model of our findings. Sumoylation of Rap1 by Ubc9 promotes RPG transcription in parallel to TORC1.

sumoylation of Rap1 mediates efficient recruitment of the basal transcription machinery.

\section{Discussion}

TFs are among the most abundant targets of Sumo, yet the global distribution of Sumo and physiological relevance of TF sumoylation are poorly understood. Here, we show that Sumo mainly localizes to pro-growth genes like tRNA genes and RPGs. The distribution of Sumo strongly resembles the genomic distribution of Sumo in mammalian cells (Neyret-Kahn et al. 2013). There have been conflicting reports as to how Sumo affects transcription at these genes in mammals; one study reported that Sumo promotes their transcription (Liu et al. 2012), whereas another study found that Sumo is inhibitory (Neyret-Kahn et al. 2013). The reason for these differences is unclear, but could be technical (Neyret-Kahn et al. 2013). These two studies did not identify the Sumo target at these genes, making it difficult to interpret their different findings. In contrast, we found that in S. cerevisiae, Sumo has a clear stimulatory effect on transcription of RPGs, and we unraveled the molecular mechanism.

We discovered that sumoylation of Rap1 is required for efficient transcription of RPGs. Sumoylation of K240/246 of Rap1 was recently shown to be important for telomere homeostasis (Lescasse et al. 2013). However, we found that preventing sumoylation of these two lysine residues, as well as various combinations of other N-terminal and C-terminal residues, had no effect on Rap1 sumoylation, RPG transcription, and cell viability. Only in the rap1-K9R mutant was sumoylation almost completely abolished, indicating that Rap1 is sumoylated on multiple lysines with (partially) redundant functions, which is commonly observed for 
Sumo and ubiquitin substrates (Fischer et al. 2011; Psakhye and Jentsch 2012). Furthermore, Rap1-K9R protein levels appeared to be increased, which could be the result of decreased protein turnover; Sumo and ubiquitin can target the same lysine residues in their substrates (Ulrich 2005), and therefore it is possible that one or several of these lysines may be involved in ubiquitin-mediated proteasomal degradation of Rap1. This is the topic of future studies.

Although Rap1 is a major Sumo target at RPG promoters, we cannot exclude the possibility that other TFs are also sumoylated. For instance, high-throughput MS studies have found that Hmo1 and Ifh1 may be Sumo targets (Wohlschlegel et al. 2004; Zhou et al. 2004). Furthermore, in addition to promoter regions, we also detected Sumo in the body of RPGs, where it likely contributes to transcription; several RPGs that contained neither Rap1 nor Sumo in their promoters did contain Sumo toward the $5^{\prime}$ end of the ORF, and their expression was reduced in the $u b c 9-1$ mutant. Here, the relevant Sumo target still needs to be identified. A good candidate is RNAPII, which was previously shown to be sumoylated; although the exact physiological significance remains unclear (Wohlschlegel et al. 2004; Zhou et al. 2004; Chen et al. 2009). Histones may also be Sumo substrates (Nathan et al. 2006). However, histone sumoylation has been associated with inhibition of transcription (Nathan et al. 2006), whereas we found that Sumo has a positive function at the majority of its target genes (Fig. 2B; Supplemental Table S3; Supplemental Fig. S2A), making it unlikely that histones are Sumo targets at these genes.

How Sumo regulates tRNA genes remains unclear. We and others have found that various components of RNAPIII, as well as mammalian Maf1 (an RNAPIII inhibitor), are Sumo substrates (Supplemental Table S5; Wohlschlegel et al. 2004; Rohira et al. 2013).

Whereas Sumo clearly activates RPGs and tDNA genes, we also found that Sumo inhibits transcription of a number of genes (Supplemental Table S3). Although this class of genes was not the focus of our study, it is worth mentioning that at least some of these genes contain binding sites for the Tup1-Ssn6 repressor complex, such as the GAL1 promoter; and sumoylation of Ssn6 was recently shown to be important for transcriptional repression of GAL1 (Texari et al. 2013). The negative effect of Sumo on transcription is the topic of future studies. Furthermore, Sumo also plays a role in chromosome duplication and DNA repair (Tempe et al. 2008), but our ChIP-seq experiments likely did not identify these events because we used unsynchronized, log-phase cells, causing the Sumo signal to disappear in the background.

Sumoylation of Rap1 stimulates its ability to recruit the basal transcription machinery. Exactly how Sumo promotes the interaction of Rap1 with TFIID is the subject of ongoing studies. Most Sumo-interacting proteins bind Sumo through low-affinity Sumo interaction motifs (SIMs) (Psakhye and Jentsch 2012). Potential SIMs are abundant in TFIID components, making the identification of functional SIMs a challenge.

In addition to recruiting TFIID, sumoylation of Rap1 may affect RPG expression through other mechanisms. For instance, Rap1 was recently shown to impact cytoplasmic decay of RPL30 mRNA (Bregman et al. 2011). Sumo levels are high at the Rap1 binding site of RPL30 (not shown). Thus, while sumoylation of Rap1 promotes RPG transcription through recruitment of TFIID, it is possible that it simultaneously restrains the expression levels of RPGs by inducing the cytoplasmic degradation of RPG mRNA.

Finally, we found that TORC1 and Ubc9 are both required for full transcription of RPGs (see Fig. 5G for a model). Paradoxically, inhibiting TORC1 resulted in increased Rap1 sumoylation at RPGs
(Fig. 2F; Supplemental Fig. S2C,E), yet inhibiting Rap1 sumoylation strongly decreased RPG transcription (Figs. 2B, 4F). Why would the cell activate the Ubc9-Sumo-Rap1 pathway under conditions that inhibit TORC1 activity? We hypothesize that the Ubc9-Sumo pathway provides positive feedback on RPG transcription to maintain cellular homeostasis. A homeostatic feedback loop has previously been reported for TORC1 (Urban et al. 2007); TORC1 activity increases when protein synthesis is inhibited by cycloheximide (Loewith and Hall 2011). Inhibiting protein synthesis with cycloheximide triggers a potent stress response (Searle et al. 1975; Tempe et al. 2008); indeed, reducing protein translation by cycloheximide induced a global increase in protein sumoylation as well as increased sumoylation of Rap1 (Supplemental Fig. S4B,C; see Supplemental Fig. S4D for a model).

In conclusion, we found that Sumo preferentially localizes to genes involved in cell growth and proliferation, and that it is required for transcription of these genes. Furthermore, Rap1 is a major target of Sumo at RPGs, and the Ubc9-Sumo-Rap1 pathway cooperates with TORC1 to promote RPG transcription. Finally, sumoylation of Rap1 stimulates RPG transcription by recruiting the basal transcription machinery.

\section{Methods}

\section{Yeast strains and plasmids}

S. cerevisiae strains were grown in appropriate media, depending on the experiment/genotype. Strains were derived directly from either the S288c strains RDKY3615 (Chen and Kolodner 1999) or BY4741 using standard gene-replacement methods or intercrossing (see Supplemental Table S6 for strains and plasmids).

\section{ChIP, ChIP-seq, and RNA-seq}

ChIP and ChIP-seq (cells fixed with 1\% [wt/vol] formaldehyde for $30 \mathrm{~min}$ ) and RNA-seq were performed as described previously (Zimmermann et al. 2011; Chymkowitch et al. 2012) with minor modifications (see Supplemental Material).

\section{Sequencing data analysis}

For all ChIP-seq and RNA-seq analyses, we used the S. cerevisiae genome sequence and associated annotation (R64-1-1.75) downloaded from Ensembl (Flicek et al. 2014). ChIP-seq reads were mapped using Bowtie 2 version 2.2.3 using default parameters (Langmead and Salzberg 2012). Generated and mapped reads are shown in Supplemental Table S1. SAM files were sorted and indexed using SAMtools (Li et al. 2009). Visualization of read density was done in the integrative genome viewer (IGV) (Robinson et al. 2011).

MACS2 version 2.0.10 was used for peak calling (Zhang et al. 2008). For Rap1 samples, automatic detection of fragment length was used. This could not be obtained for Sumo; instead, a fragment length of 200 was used. We filtered peaks called by MACS2 using control samples with no tag to identify a cutoff at which few peaks were called in the control samples. For Rap1, a $\log _{10} P$-value of 50 was used. Still, a few peaks remained in the control sample, which we filtered out unless they had a twice as high score in the ChIPsample. BEDTools (Quinlan and Hall 2010) was used to identify overlapping peaks, and for all intersections, a 1-bp overlap requirement was used. For analysis of the Sumo ChIP-seq data, a $\log _{10} P$ value of 6 was used, resulting in a data set of 631 genes (Supplemental Table S1). Peaks were then annotated according to

\section{Genome Research}

www.genome.org 
genomic location and the closest overlapping gene using ChIPpeakAnno (Zhu et al. 2010) and biomaRt (Durinck et al. 2009). We used the middle of the peak to measure the distance to TSSs. Manual inspection of the resulting Sumo ChIP-seq data set revealed a significant number of ORFs with relatively low Sumo signals. These were absent when the data set was filtered with a more stringent $\log _{10} P$-value of 20 . However, this stringent data set still contained several ORFs (particularly dubious/hypothetical ORFs) that were in close proximity to either tRNA genes or promoter regions of ORFs with high Sumo levels, especially RPGs. In most of these cases, visual inspection revealed that the software had improperly assigned Sumo peaks to the wrong ORF, because the algorithm assigns the peak to the TSS of the ORF that is most closely positioned to the summit of the peak, which is incorrect in a minority of cases (e.g., YGR164W, YDR448W, YDR022C, YBR085W, YPR131C, and YMR196C-A) (see Supplemental Fig. S1C). These overlapping dubious/hypothetical ORFs were removed from the data set, whereas the genes that were originally not called by the software due to such wrongful assignment were added back to the data set [for the examples shown in Supplemental Fig. S1C, these were $t R(U C U) G 2, R P S 17 B, t V(U A C) D$, RPL19A, RPS23B, and RPL36A]. This resulted in a high-confidence data set consisting of a total number of 395 genes that are highly enriched for Sumo (Supplemental Table S1).

Functional annotation of associated genes was performed in the functional annotation tool found at http://www. yeastgenome.org/. See Supplemental information for further details.

\section{RNA preparation and reverse transcription}

Total RNA purification and reverse transcription were performed as previously described (Chymkowitch et al. 2012).

\section{Sumo pull-down under denaturing conditions}

Pull-downs in denaturing conditions were performed as previously described (Sacher et al. 2005) with minor modifications (see Supplemental Experimental Procedures).

\section{Cell lysis, immunoprecipitation, and Western blotting}

IP and Western blotting was performed as previously described (Sacher et al. 2005; Kats et al. 2009; Chymkowitch et al. 2012). All buffers contained N-Ethylmaleimide to prevent desumoylation.

\section{Data access}

All high-throughput sequencing data have been submitted to the European Nucleotide Archive (ENA; http://www.ebi.ac.uk/ena). See Supplemental Table S7 for sample accession numbers. The ChIP-seq study accession number is PRJEB7579, and the study unique name is ena-STUDY-Oslo Genomics Core Facility-21-102014-13:44:44:043-43. The RNA-seq study accession number is PRJEB7579, and the study unique name is ena-STUDY-Oslo Genomics Core Facility-21-10-2014-13:44:44:043-43.

\section{Acknowledgments}

We gratefully acknowledge Drs. W. Seufert, H. Zhou, and S. Alberti for reagents; M. Bjørås and P. Collas for infrastructure and discussions; and Dr. J. Krijgsveld for mass spectrometry services. This project is supported by grants to J.M.E. from the Norwegian Research Council (221694) and the Norwegian Cancer Society (project number 3311782). P.C. is supported by a grant from the Norwegian Research Council (221920).

Author contributions: J.M.E. conceived the project. P.C., A.N.P., and J.M.E. designed and performed experiments. Mass spectrometry was performed by C.J.K. H.A. and S.L. performed computational analysis of genome-wide data sets. P.C. and J.M.E. wrote the paper with input from all other authors.

\section{References}

Albuquerque CP, Wang G, Lee NS, Kolodner RD, Putnam CD, Zhou H. 2013. Distinct SUMO ligases cooperate with Esc2 and Slx5 to suppress duplication-mediated genome rearrangements. PLoS Genet 9: e1003670.

Betting J, Seufert W. 1996. A yeast Ubc9 mutant protein with temperaturesensitive in vivo function is subject to conditional proteolysis by a ubiquitin- and proteasome-dependent pathway. I Biol Chem 271: 25790-25796.

Bregman A, Avraham-Kelbert M, Barkai O, Duek L, Guterman A, Choder M. 2011. Promoter elements regulate cytoplasmic mRNA decay. Cell 147: 1473-1483.

Cai L, McCormick MA, Kennedy BK, Tu BP. 2013. Integration of multiple nutrient cues and regulation of lifespan by ribosomal transcription factor Ifh1. Cell Rep 4: 1063-1071.

Chen C, Kolodner RD. 1999. Gross chromosomal rearrangements in Saccharomyces cerevisiae replication and recombination defective mutants. Nat Genet 23: 81-85.

Chen X, Ding B, LeJeune D, Ruggiero C, Li S. 2009. Rpb1 sumoylation in response to UV radiation or transcriptional impairment in yeast. PLoS One 4: e5267.

Chymkowitch P, Eldholm V, Lorenz S, Zimmermann C, Lindvall JM, Bjørås M, Meza-Zepeda LA, Enserink JM. 2012. Cdc28 kinase activity regulates the basal transcription machinery at a subset of genes. Proc Natl Acad Sci 109: 10450-10455.

De Virgilio C, Loewith R. 2006. Cell growth control: little eukaryotes make big contributions. Oncogene 25: 6392-6415.

Denison C, Rudner AD, Gerber SA, Bakalarski CE, Moazed D, Gygi SP. 2005. A proteomic strategy for gaining insights into protein sumoylation in yeast. Mol Cell Proteomics 4: 246-254.

Durinck S, Bullard J, Spellman PT, Dudoit S. 2009. GenomeGraphs: integrated genomic data visualization with R. BMC Bioinformatics 10: 2 .

Fischer ES, Scrima A, Böhm K, Matsumoto S, Lingaraju GM, Faty M, Yasuda T, Cavadini S, Wakasugi M, Hanaoka F, et al. 2011. The molecular basis of CRL $4^{\mathrm{DDB} 2 / \mathrm{CSA}}$ ubiquitin ligase architecture, targeting, and activation. Cell 147: 1024-1039.

Flicek P, Amode MR, Barrell D, Beal K, Billis K, Brent S, Carvalho-Silva D, Clapham P, Coates G, Fitzgerald S, et al. 2014. Ensembl 2014. Nucleic Acids Res 42: D749-D755.

Garbett KA, Tripathi MK, Cencki B, Layer JH, Weil PA. 2007. Yeast TFIID serves as a coactivator for Rap1p by direct protein-protein interaction. Mol Cell Biol 27: 297-311.

Gill G. 2005. Something about SUMO inhibits transcription. Curr Opin Genet Dev 15: 536-541.

Hang LE, Liu X, Cheung I, Yang Y, Zhao X. 2011. SUMOylation regulates telomere length homeostasis by targeting Cdc13. Nat Struct Mol Biol 18: 920-926.

Hannich JT, Lewis A, Kroetz MB, Li SJ, Heide H, Emili A, Hochstrasser M. 2005. Defining the SUMO-modified proteome by multiple approaches in Saccharomyces cerevisiae. J Biol Chem 280: 4102-4110.

Jentsch S, Psakhye I. 2013. Control of nuclear activities by substrate-selective and protein-group SUMOylation. Annu Rev Genet 47: 167-186.

Johnson ES, Blobel G. 1999. Cell cycle-regulated attachment of the ubiquitin-related protein SUMO to the yeast septins. J Cell Biol 147: 981-994.

Kats ES, Enserink JM, Martinez S, Kolodner RD. 2009. The Saccharomyces cerevisiae Rad6 post replication repair and Siz1/Srs2 homologous recombination-inhibiting pathways process DNA damage that arises in asf1 mutants. Mol Cell Biol 29: 5226-5237.

Knight B, Kubik S, Ghosh B, Bruzzone MJ, Geertz M, Martin V, Dénervaud N, Jacquet P, Ozkan B, Rougemont J, et al. 2014. Two distinct promoter architectures centered on dynamic nucleosomes control ribosomal protein gene transcription. Genes Dev 28: 1695-1709.

Langmead B, Salzberg SL. 2012. Fast gapped-read alignment with Bowtie 2. Nat Methods 9: 357-359.

Lempiainen H, Shore D. 2009. Growth control and ribosome biogenesis. Curr Opin Cell Biol 21: 855-863.

Lescasse R, Pobiega S, Callebaut I, Marcand S. 2013. End-joining inhibition at telomeres requires the translocase and polySUMO-dependent ubiquitin ligase Uls1. EMBO J 32: 805-815. 
Li H, Handsaker B, Wysoker A, Fennell T, Ruan J, Homer N, Marth G, Abecasis G, Durbin R; 1000 Genome Project Data Processing Subgroup. 2009. The Sequence Alignment/Map format and SAMtools. Bioinformatics 25: 2078-2079.

Lickwar CR, Mueller F, Hanlon SE, McNally JG, Lieb JD. 2012. Genome-wide protein-DNA binding dynamics suggest a molecular clutch for transcription factor function. Nature 484: 251-255.

Lieb JD, Liu X, Botstein D, Brown PO. 2001. Promoter-specific binding of Rap1 revealed by genome-wide maps of protein-DNA association. Nat Genet 28: 327-334.

Liu HW, Zhang J, Heine GF, Arora M, Gulcin Ozer H, Onti-Srinivasan R, Huang K, Parvin JD. 2012. Chromatin modification by SUMO-1 stimulates the promoters of translation machinery genes. Nucleic Acids Res 40: 10172-10186.

Loewith R, Hall MN. 2011. Target of rapamycin (TOR) in nutrient signaling and growth control. Genetics 189: 1177-1201.

Loewith R, Jacinto E, Wullschleger S, Lorberg A, Crespo JL, Bonenfant D, Oppliger W, Jenoe P, Hall MN. 2002. Two TOR complexes, only one of which is rapamycin sensitive, have distinct roles in cell growth control. Mol Cell 10: 457-468.

Nathan D, Ingvarsdottir K, Sterner DE, Bylebyl GR, Dokmanovic M, Dorsey JA, Whelan KA, Krsmanovic M, Lane WS, Meluh PB, et al. 2006. Histone sumoylation is a negative regulator in Saccharomyces cerevisiae and shows dynamic interplay with positive-acting histone modifications. Genes Dev 20: 966-976.

Neyret-Kahn H, Benhamed M, Ye T, Le Gras S, Cossec JC, Lapaquette P, Bischof O, Ouspenskaia M, Dasso M, Seeler J, et al. 2013. Sumoylation at chromatin governs coordinated repression of a transcriptional program essential for cell growth and proliferation. Genome Res 23: 1563-1579.

Psakhye I, Jentsch S. 2012. Protein group modification and synergy in the SUMO pathway as exemplified in DNA repair. Cell 151: 807-820.

Quinlan AR, Hall IM. 2010. BEDTools: a flexible suite of utilities for comparing genomic features. Bioinformatics 26: 841-842.

Robinson JT, Thorvaldsdóttir H, Winckler W, Guttman M, Lander ES, Getz G, Mesirov JP. 2011. Integrative genomics viewer. Nat Biotechnol 29: 24-26.

Rohira AD, Chen CY, Allen JR, Johnson DL. 2013. Covalent small ubiquitinlike modifier (SUMO) modification of Maf1 protein controls RNA polymerase III-dependent transcription repression. I Biol Chem 288: 19288-19295.

Rosonina E, Duncan SM, Manley JL. 2010. SUMO functions in constitutive transcription and during activation of inducible genes in yeast. Genes Dev 24: 1242-1252.

Sacher M, Pfander B, Jentsch S. 2005. Identification of SUMO-protein conjugates. Methods Enzymol 399: 392-404.

Schawalder SB, Kabani M, Howald I, Choudhury U, Werner M, Shore D. 2004. Growth-regulated recruitment of the essential yeast ribosomal protein gene activator Ifh1. Nature 432: 1058-1061.

Searle J, Lawson TA, Abbott PJ, Harmon B, Kerr JF. 1975. An electron-microscope study of the mode of cell death induced by cancer-chemothera- peutic agents in populations of proliferating normal and neoplastic cells. J Pathol 116: 129-138.

Shore D. 1997. Telomere length regulation: getting the measure of chromosome ends. Biol Chem 378: 591-597.

Simpson CE, Ashe MP. 2012. Adaptation to stress in yeast: to translate or not? Biochem Soc Trans 40: 794-799.

Tempe D, Piechaczyk M, Bossis G. 2008. SUMO under stress. Biochem Soc Trans 36: 874-878.

Texari L, Dieppois G, Vinciguerra P, Contreras MP, Groner A, Letourneau A Stutz F. 2013. The nuclear pore regulates GAL1 gene transcription by controlling the localization of the SUMO protease Ulp1. Mol Cell 51: 807-818.

Teytelman L, Thurtle DM, Rine J, van Oudenaarden A. 2013. Highly expressed loci are vulnerable to misleading ChIP localization of multiple unrelated proteins. Proc Natl Acad Sci 110: 18602-18607.

Ulrich HD. 2005. Mutual interactions between the SUMO and ubiquitin systems: a plea of no contest. Trends Cell Biol 15: 525-532.

Urban J, Soulard A, Huber A, Lippman S, Mukhopadhyay D, Deloche O, Wanke V, Anrather D, Ammerer G, Riezman H, et al. 2007. Sch9 is a major target of TORC1 in Saccharomyces cerevisiae. Mol Cell 26: 663-674.

Wade JT, Hall DB, Struhl K. 2004. The transcription factor Ifh1 is a key regulator of yeast ribosomal protein genes. Nature 432: 1054-1058.

Warner JR. 1999. The economics of ribosome biosynthesis in yeast. Trends Biochem Sci 24: 437-440.

Wei Y, Zheng XS. 2010. Maf1 regulation: a model of signal transduction inside the nucleus. Nucleus 1: 162-165.

Wohlschlegel JA, Johnson ES, Reed SI, Yates JR III. 2004. Global analysis of protein sumoylation in Saccharomyces cerevisiae. J Biol Chem 279: $45662-45668$.

Zhang Y, Liu T, Meyer CA, Eeckhoute J, Johnson DS, Bernstein BE, Nusbaum C, Myers RM, Brown M, Li W, et al. 2008. Model-based analysis of ChIPSeq (MACS). Genome Biol 9: R137.

Zhao Q, Xie Y, Zheng Y, Jiang S, Liu W, Mu W, Liu Z, Zhao Y, Xue Y, Ren J. 2014. GPS-SUMO: a tool for the prediction of sumoylation sites and SUMO-interaction motifs. Nucleic Acids Res 42: W325-W330.

Zhou W, Ryan JJ, Zhou H. 2004. Global analyses of sumoylated proteins in Saccharomyces cerevisiae. Induction of protein sumoylation by cellular stresses. J Biol Chem 279: 32262-32268.

Zhu LJ, Gazin C, Lawson ND, Pagès H, Lin SM, Lapointe DS, Green MR. 2010. ChIPpeakAnno: a Bioconductor package to annotate ChIP-sec and ChIP-chip data. BMC Bioinformatics 11: 237.

Zimmermann C, Chymkowitch P, Eldholm V, Putnam CD, Lindvall JM, Omerzu M, Bjørås M, Kolodner RD, Enserink JM. 2011. A chemical-genetic screen to unravel the genetic network of CDC28/CDK1 links ubiquitin and Rad6-Bre1 to cell cycle progression. Proc Natl Acad Sci 108: 18748-18753.

Received October 13, 2014; accepted in revised form March 17, 2015.

\section{Genome Research}

www.genome.org 


\section{Corrigendum}

Genome Research 25: 897-906 (2015)

\section{Corrigendum: Sumoylation of Rap1 mediates the recruitment of TFIID to promote transcrip- tion of ribosomal protein genes}

Pierre Chymkowitch, Aurélie Nguéa P, Håvard Aanes, Christian J. Koehler, Bernd Thiede, Susanne Lorenz, Leonardo A. Meza-Zepeda, Arne Klungland, and Jorrit M. Enserink

The authors would like to correct Figure 4 panel D, in which one negative control panel (i.e., the fourth panel of the middle row) was inadvertently duplicated during manuscript preparation. This panel displays a spot assay of cells expressing RAP1 from its endogenous promoter and transformed with either an empty vector, or with plasmids containing either RAP1, rap1-K3NR, rap1-K3CR, rap1-K9R, or rap1-K651R under control of the GPD promoter on plates containing glucose, and grown at $37^{\circ} \mathrm{C}$ until colonies appeared. The corrected Figure 4D is provided below. This correction does not alter the interpretation of the figure or the conclusions of the article. The authors apologize for any confusion this may have caused.

D
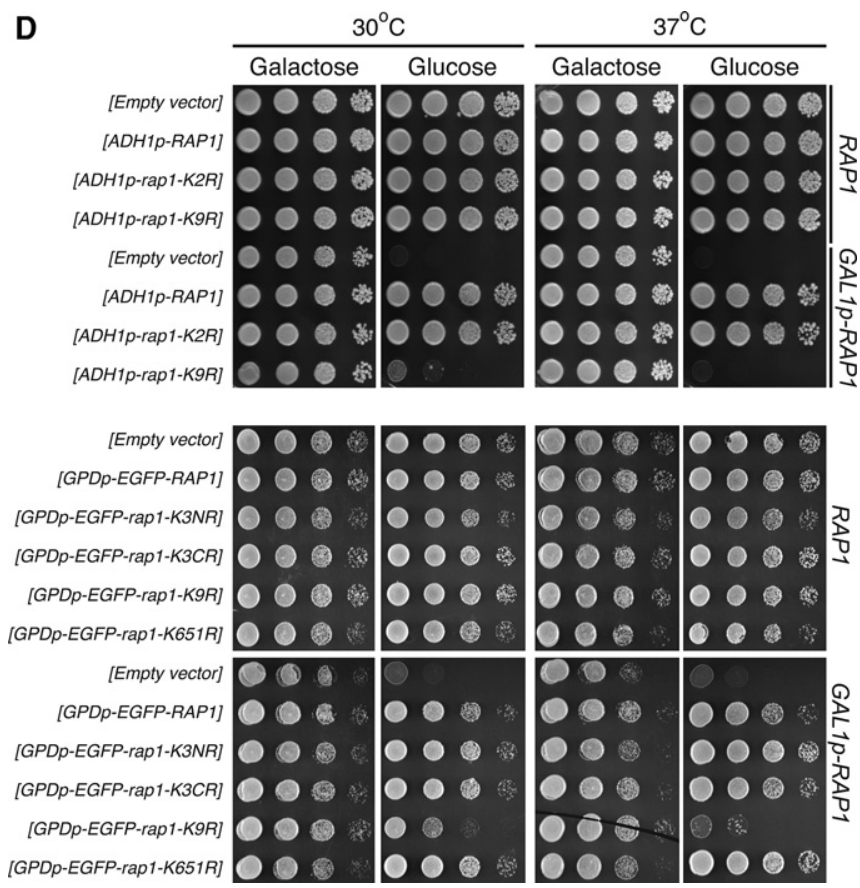

Figure 4D. 


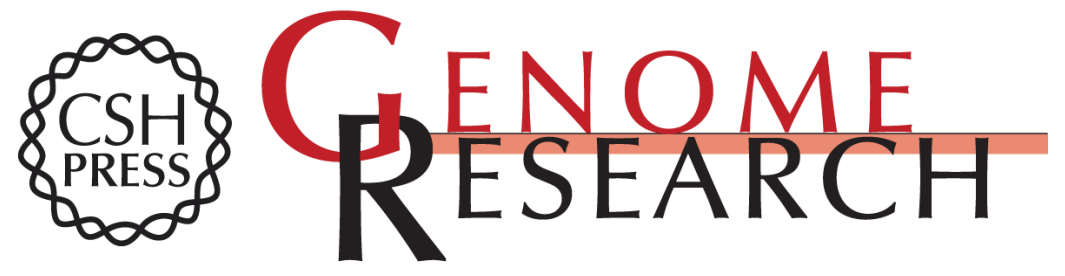

\section{Sumoylation of Rap1 mediates the recruitment of TFIID to promote transcription of ribosomal protein genes}

Pierre Chymkowitch, Aurélie Nguéa P, Håvard Aanes, et al.

Genome Res. 2015 25: 897-906 originally published online March 23, 2015

Access the most recent version at doi:10.1101/gr.185793.114

\section{Supplemental http://genome.cshlp.org/content/suppl/2015/04/16/gr.185793.114.DC1 \\ Material}
Related Content Corrigendum: Sumoylation of Rap1 mediates the recruitment of TFIID to promote transcription of ribosomal protein genes
Pierre Chymkowitch, Aurélie Nguéa $P$, Håvard Aanes, et al.
Genome Res. February, 2017 27: 334

References This article cites 55 articles, 20 of which can be accessed free at:

http://genome.cshlp.org/content/25/6/897.full.html\#ref-list-1

Articles cited in:

http://genome.cshlp.org/content/25/6/897.full.htmI\#related-urls

Open Access Freely available online through the Genome Research Open Access option.

Creative This article, published in Genome Research, is available under a Creative

Commons Commons License (Attribution-NonCommercial 4.0 International), as described at

License http://creativecommons.org/licenses/by-nc/4.0/.

Email Alerting Receive free email alerts when new articles cite this article - sign up in the box at the Service top right corner of the article or click here.

\section{Affordable, Accurate Sequencing.}

